\title{
Indium(III) Trifluoromethanesulfonate: a Reusable Catalyst for the Sol- vent-free Synthesis of some Quinazolinones/thiones
}

\author{
Bahador Karami, ${ }^{a, *}$ Saeed Khodabakhshi, ${ }^{\text {b and Sajad Karami }}{ }^{a}$ \\ ${ }^{a}$ Department of Chemistry, Gachsaran Branch, Islamic Azad University, Gachsaran, Iran \\ ${ }^{\mathrm{b}}$ Young Researchers and Elite Club, Gachsaran Branch, Islamic Azad University, Gachsaran, Iran
}

RECEIVED MARCH 12, 2012; REVISED OCTOBER 16, 2013; ACCEPTED NOVEMBER 13, 2013

\begin{abstract}
An environmentally friendly and clean procedure gives octahydroquinazolinones/thiones by a simple Biginelli condensation of urea/thiourea, aromatic aldehydes, and cyclic 1,3-diones in the presence of indium(III) trifluoromethanesulfonate $\left(\operatorname{In}(\mathrm{OTf})_{3}\right)$ in solventless conditions. This method has advantages such as avoidance of the organic solvents, high yield of pure products without any by-product, short reaction times and operational simplicity.(doi: 10.5562/cca2087)
\end{abstract}

Keywords: octahydroquinazolinones/thiones, indium (III) trifluoromethanesulfonate, urea/thiourea, aldehyde

\section{INTRODUCTION}

Recently, pressure from environmentalists has led to a search for more environmentally friendly forms of catalysis. ${ }^{1,2}$ A wide variety of Lewis acids have been used for organic transformations. ${ }^{3}$ Most of the classical reagents of this class, such as $\mathrm{AlCl}_{3}, \mathrm{BF}_{3} / \mathrm{BF}_{3} \cdot \mathrm{Et}_{2} \mathrm{O}, \mathrm{FeCl}_{3}$, $\mathrm{FeCl}_{2}, \mathrm{TiCl}_{4}$, and $\mathrm{ZnCl}_{2}$, are extremely sensitive to moisture and are difficult to handle. Besides, they are often used in stoichiometric quantities for the reactions. In the last two decades, multicomponent reactions (MCRs) have drawn special attention due to the advent of high-throughput screening techniques that enabled rapid identification of potential new medicines among large collections of organic compounds. ${ }^{4,5}$ Moreover; the chemistry of quinazolin system has received an increasing interest because of its biological significance. They are a class of drugs which function as hypnotic/sedatives. For example, the Afloqualone, Cloroqualone, and Diproqualone (Figure 1) have also been used in the treatment of cancer. ${ }^{6}$

The most general method for the preparation of octahydroquinazolinones/thiones involves the one-pot Biginelli condensation reaction of cyclic 1,3-dione, aromatic aldehydes and urea/thiourea in the presence of a Lewis or mineral acids. Although, synthesis of octahydroquinazolinones/thiones via the Biginelli reaction using several reagents have been previously reported, ${ }^{7-14}$ some of the procedures have disadvantages such as, long reaction time, use of strongly acidic condition or organic solvents, unsatisfactory product yield, and also side products. Due to its low toxicity, ease of han-

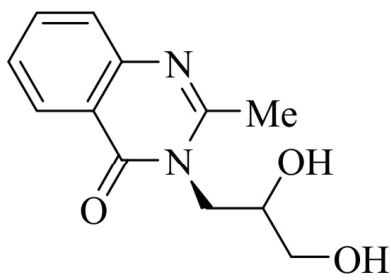

Diproqualone

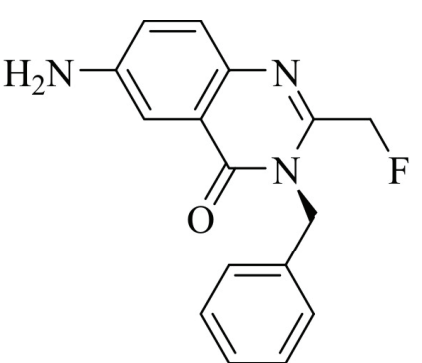

Afloqualone<smiles>CCc1nc2ccccc2c(=O)n1-c1c(Cl)cccc1Cl</smiles>

Cloroqualone

Figure 1. Quinazolinene derivatives as anti cancer compounds

\footnotetext{
* Author to whom correspondence should be addressed. (E-mail: karami@mail.yu.ac.ir)
} 


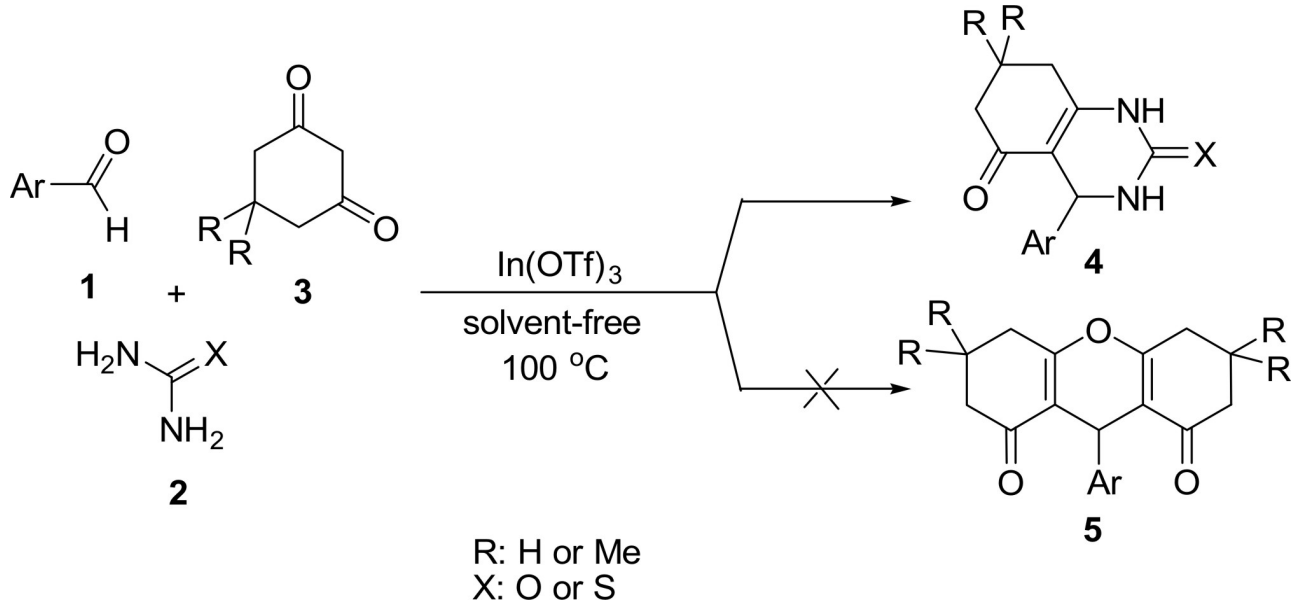

Scheme 1. Three-component condensation of arylaldehydes, cyclic 1,3-dione, and urea/thiourea using $\operatorname{In}(\mathrm{OTf})_{3}$.

dling, and high activity, the In(III) triflate has emerged as a useful catalyst in many organic synthesis, including, highly chemo and regioselective head-to-tail heterodimerization of vinylarenes, ${ }^{15}$ chemoselective conversion of aldehydes to acylals, ${ }^{16}$ synthesis of 2,4disubstituted quinolines, ${ }^{17}$ reaction of sugars, ${ }^{18}$ and Friedel-Crafts sulfonylation of arenas. ${ }^{19}$ To the best of our knowledge no work has been reported in the literature on the use of In(III) triflate for the Biginelli condensation. Therefore, an investigation was carried out in order to explore its catalytic application in the Biginelli condensation and the results are reported herein.

\section{RESULTS AND DISCUSSION}

In connection with our previous programs on synthesis of organic compounds, ${ }^{20-23}$ in this work, we wish to report a simple and convenient synthesis of octahydroquinazolinones/thiones 4 by reaction of aromatic aldehyde $\mathbf{1}$, urea/thiourea $\mathbf{2}$, and cyclic 1,3-dione $\mathbf{3}$, in the presence of catalytic amount of $\operatorname{In}(\mathrm{OTf})_{3}$ under solvent-free conditions (Scheme 1). The yield of products was good to excellent without the formation of octahydoxanthenes $\mathbf{5}$, which is the major product of the procedure reported by the literature. ${ }^{24}$

The need to implement green chemistry principles (e.g. safer solvents, less hazardous chemical synthesis, atom economy, and catalysis) is a driving force towards the avoidance of organic solvents and use of environmentally friendly, and reusable catalysts. A solvent-free or solid state reaction obviously reduce pollution, and bring down handling costs due to simplification of experimental procedure, work up technique and saving in labour. However, interest in the environmental control of chemical processes has increased remarkably during three decades ago as a response to public concern about the use of hazardous chemicals. Therefore, to improve the effectiveness of this method in preventing chemical waste, it is important to investigate optimal reaction conditions.

To find the simple and suitable conditions for the preparation of 4 using $\operatorname{In}(\mathrm{OTf})_{3}$ as a powerful Lewis acid catalyst, the treatment of benzaldehyde, dimedone, and urea was chosen as a model reaction. At first, we found that in the absence of $\operatorname{In}(\mathrm{OTf})_{3}$, the reaction did not proceed even at a high temperature after long reaction time. After examining the various amounts of $\mathrm{In}(\mathrm{OTf})_{3}$ and a wide range of temperatures (Table 1), we found that this reaction can be efficiently carried out by adding $5 \mathrm{~mol} \%$ of the catalyst at $100{ }^{\circ} \mathrm{C}$ under solventfree conditions in a short time span of $45 \mathrm{~min}$. The use of excessive amounts of the catalyst does not increase the product yield or reaction rate.

The scope of this MCR was examined using a variety of aromatic aldehydes. It was found that both, electron rich and electron poor aldehydes reacted well in this process to afford the corresponding products $\mathbf{4}$ in

Table 1. Optimization of reaction conditions

\begin{tabular}{cccc}
\hline Entry & $\begin{array}{c}\text { Catalyst }\left(\mathrm{mol}^{\%} \%\right) / \\
\text { Temperature }\left({ }^{\circ} \mathrm{C}\right)\end{array}$ & Time / min & Yield $^{(\mathrm{a})} / \%$ \\
\hline 1 & $-/ 25$ & 380 & trace \\
2 & $-/ 60$ & 380 & 20 \\
3 & $-/ 100$ & 380 & 35 \\
4 & $-/ 150$ & 380 & 25 \\
5 & $3 / 25$ & 380 & 50 \\
6 & $3 / 100$ & 260 & 70 \\
7 & $3 / 120$ & 260 & 70 \\
8 & $5 / 25$ & 380 & 40 \\
9 & $5 / 60$ & 380 & 70 \\
10 & $5 / 80$ & 260 & 80 \\
11 & $5 / 100$ & 45 & 90 \\
12 & $5 / 120$ & 40 & 80 \\
13 & $10 / 100$ & 65 & 90 \\
14 & $10 / 120$ & 55 & 75 \\
15 & $15 / 100$ & 80 & 90 \\
\hline
\end{tabular}

\footnotetext{
${ }^{(a)}$ Isolated yields
} 
Table 2. Synthesis of 4 via the Biginelli method using $\operatorname{In}(\mathrm{OTf})_{3}$ at $100{ }^{\circ} \mathrm{C}$ under solvent-free conditions

\begin{tabular}{|c|c|c|c|c|c|c|}
\hline Entry & $\mathrm{R}$ & $\mathrm{Ar}$ & $\mathrm{X}$ & Time / min & Yield $^{(\mathrm{a})} / \%$ & M.p. $\left({ }^{\circ} \mathrm{C}\right)$ [lit.] \\
\hline $4 a$ & $\mathrm{Me}$ & $\mathrm{C}_{6} \mathrm{H}_{5}$ & $\mathrm{O}$ & 45 & 90 & $288-290$ [7] \\
\hline $4 b$ & $\mathrm{Me}$ & $4-\mathrm{MeO}-\mathrm{C}_{6} \mathrm{H}_{4}$ & $\mathrm{O}$ & 50 & 80 & $272-274[8]$ \\
\hline $4 c$ & $\mathrm{Me}$ & $2-\mathrm{Cl}-\mathrm{C}_{6} \mathrm{H}_{4}$ & $\mathrm{O}$ & 50 & 85 & $271-273[7]$ \\
\hline 4d & $\mathrm{Me}$ & 4-Br- $\mathrm{C}_{6} \mathrm{H}_{4}$ & $\mathrm{O}$ & 30 & 80 & $324-326$ [9] \\
\hline $4 f$ & $\mathrm{Me}$ & $4-\mathrm{F}-\mathrm{C}_{6} \mathrm{H}_{4}$ & $\mathrm{O}$ & 40 & 78 & $300-302$ [7] \\
\hline $4 g$ & $\mathrm{Me}$ & 2-MeO- $\mathrm{C}_{6} \mathrm{H}_{4}$ & $\mathrm{O}$ & 30 & 85 & 197-199 [7] \\
\hline $4 \mathrm{~h}$ & $\mathrm{Me}$ & $4-\mathrm{Me}-\mathrm{C}_{6} \mathrm{H}_{4}$ & $\mathrm{O}$ & 30 & 90 & $300-302$ [9] \\
\hline $4 i$ & $\mathrm{Me}$ & $3-\mathrm{O}_{2} \mathrm{~N}-\mathrm{C}_{6} \mathrm{H}_{4}$ & $\mathrm{O}$ & 25 & 90 & $297-299$ [10] \\
\hline $4 j$ & $\mathrm{H}$ & $\mathrm{C}_{6} \mathrm{H}_{5}$ & $\mathrm{O}$ & 40 & 90 & $275-277[11]$ \\
\hline $4 \mathbf{k}$ & $\mathrm{H}$ & $4-\mathrm{Cl}-3-\mathrm{O}_{2} \mathrm{~N}-\mathrm{C}_{6} \mathrm{H}_{3}$ & $\mathrm{O}$ & 30 & 85 & $209-211$ [13] \\
\hline 41 & $\mathrm{H}$ & 4-Br- $\mathrm{C}_{6} \mathrm{H}_{4}$ & $\mathrm{O}$ & 35 & 80 & $275-277[8]$ \\
\hline $4 m$ & $\mathrm{H}$ & $4-\mathrm{Cl}-\mathrm{C}_{6} \mathrm{H}_{4}$ & $\mathrm{O}$ & 30 & 85 & $281-282[11]$ \\
\hline $4 n$ & $\mathrm{H}$ & $2-\mathrm{MeO}-\mathrm{C}_{6} \mathrm{H}_{4}$ & $\mathrm{O}$ & 50 & 80 & $197-199$ [12] \\
\hline 40 & $\mathrm{Me}$ & $2,4-\mathrm{Cl}_{2}-\mathrm{C}_{6} \mathrm{H}_{3}$ & $\mathrm{O}$ & 25 & 85 & $263-265[10]$ \\
\hline $4 p$ & $\mathrm{Me}$ & $3-\mathrm{Br}-\mathrm{C}_{6} \mathrm{H}_{4}$ & $\mathrm{O}$ & 20 & 90 & $265-267$ [23] \\
\hline $4 q$ & $\mathrm{Me}$ & $3-\mathrm{Cl}-\mathrm{C}_{6} \mathrm{H}_{4}$ & $\mathrm{O}$ & 40 & 80 & $290-292$ [23] \\
\hline $4 r$ & $\mathrm{Me}$ & $4-\mathrm{Me}-\mathrm{C}_{6} \mathrm{H}_{4}$ & $\mathrm{~S}$ & 35 & 85 & $280-282[10]$ \\
\hline $4 s$ & $\mathrm{Me}$ & $4-\mathrm{Br}-\mathrm{C}_{6} \mathrm{H}_{4}$ & $\mathrm{~S}$ & 70 & 75 & $290-292$ [20] \\
\hline $4 t$ & $\mathrm{Me}$ & $4-\mathrm{MeO}-\mathrm{C}_{6} \mathrm{H}_{4}$ & $\mathrm{~S}$ & 60 & 80 & $268-270$ [12] \\
\hline $4 u$ & $\mathrm{Me}$ & $\mathrm{C}_{6} \mathrm{H}_{5}$ & $\mathrm{~S}$ & 75 & 80 & $280-282[12]$ \\
\hline
\end{tabular}

(a) Isolated yields

good to excellent yields. The obtained results summarized in Table 2.

The suggested mechanism for the formation of $\mathbf{4}$ using $\operatorname{In}(\mathrm{OTf})_{3}$ is shown in Scheme 2. Activation of the carbonyl groups by Lewis acid leads to Knoevenagel condensation between $\beta$-diketon and aldehyde. After that, 1,4-addition of nucleophile (urea/thiourea) to the $\alpha, \beta$-unsaturated ketone followed by intramolecular cyclization and dehydration generates the target molecule 4.

In view of eco-friendly procedure, the recovery and reuse of this catalyst is quite preferable. Indium(III) trifluoromethanesulfonate was easily separated from the reaction mixture by filtering, followed by drying at room temperature. The catalyst was reused three times for synthesis of $\mathbf{4 a}$ without significant loose of activity.

\section{EXPERIMENTAL SECTION}

\section{General}

All chemicals were purchased from Merck, Fluka and Aldrich. The reactions were monitored by TLC (silicagel $60 \mathrm{~F}_{254}$, hexane/EtOAc). IR spectra were recorded<smiles>[X]C1=NC([Al])C2=C(CC(C)(C)CC2=O)NC([X])N1</smiles>

Scheme 2. Suggested mechanism for the synthesis of 4 with $\operatorname{In}(\mathrm{OTf})_{3}$. 
on a FT-IR JASCO-680 and the NMR spectra were obtained on a Bruker-Instrument DPX-400 MHz Avance 2 model. The varioEl CHNS Isfahan Industrial University was used for elemental analysis. The melting point of all synthesized compounds was compared with those reported in literature and they are in agreement.

\section{General Procedure for the Preparation of Octahy- droquinazolinones/thiones}

A mixture of urea/thiourea $(1.2 \mathrm{mmol})$, aldehyde (1 $\mathrm{mmol})$, cyclic 1,3-dione $(1 \mathrm{mmol})$, and $\operatorname{In}(\mathrm{OTf})_{3}$ (5 mol\%) was stirred and heated at $100{ }^{\circ} \mathrm{C}$ in a preheated oil bath for an appropriate time (Table 1). After completion of the reaction as indicated by TLC, the reaction mixture was cooled to room temperature and THF (10 $\mathrm{mL}$ ) was added. Then the resulting mixture was stirred for $3 \mathrm{~min}$. The catalyst was separated by filtration. The solvent was removed by distillation, then washed with cold water and recrystallized from methanol to afford the pure product.

\section{Selected Spectral Data}

4b: IR (KBr) $v_{\max } / \mathrm{cm}^{-1}: 3285$ (s), 3200 (s), 1640 (s), 1605 (s); ${ }^{1} \mathrm{H}$ NMR (DMSO- $\left.d_{6}, 400 \mathrm{MHz}\right) \delta / \mathrm{ppm}: 0.91$ $\left(3 \mathrm{H}, \mathrm{s}, \mathrm{CH}_{3}\right), 1.04\left(3 \mathrm{H}, \mathrm{s}, \mathrm{CH}_{3}\right), 1.97-2.01(2 \mathrm{H}, \mathrm{d}, J=$ $\left.16 \mathrm{~Hz}, \mathrm{CH}_{2}\right), 2.17-2.19\left(2 \mathrm{H}, \mathrm{d}, J=8 \mathrm{~Hz}, \mathrm{CH}_{2}\right)$, 2.30-2.34 (2H, d, $\left.J=16 \mathrm{~Hz}, \mathrm{CH}_{2}\right), 2.47-2.51(2 \mathrm{H}, \mathrm{d}, J$ $\left.=16 \mathrm{~Hz}, \mathrm{CH}_{2}\right), 3.66\left(3 \mathrm{H}, \mathrm{s}, \mathrm{OCH}_{3}\right), 4.76(1 \mathrm{H}, \mathrm{s}, \mathrm{CH})$, 6.86-7.07 (m, 4H, Arom), $7.76(1 \mathrm{H}, \mathrm{s}, \mathrm{NH}), 9.27(1 \mathrm{H}$, $\mathrm{s}, \mathrm{NH}) \mathrm{ppm} ;{ }^{13} \mathrm{C}$ NMR (DMSO- $\left.d_{6}, 100 \mathrm{MHz}\right) \delta / \mathrm{ppm}$ : $27.34\left(\mathrm{CH}_{3}\right), 29.16\left(\mathrm{CH}_{3}\right), 31.56(\mathrm{C}), 32.75\left(\mathrm{CH}_{2}\right)$, $50.52\left(\mathrm{CH}_{2}\right), 51.84(\mathrm{CH}), 55.29\left(\mathrm{OCH}_{3}\right), 107.84(\mathrm{C})$, $113.39(\mathrm{CH}), 128.99(\mathrm{CH}), 135.15(\mathrm{C}), 149.52(\mathrm{C})$, 153.61 (C), 157.55 (CO), 194.86 (CO) ppm; Anal. Calcd. for $\mathrm{C}_{17} \mathrm{H}_{20} \mathrm{~N}_{2} \mathrm{O}_{3}$ : C, 67.98; H, 6.71; N, $9.33 \%$. Found: C, 68.23; H, 6.41; N, 9.25\%.

4c: IR (KBr) $v_{\max } / \mathrm{cm}^{-1}: 3443$ (s), 3192 (s), 1681 (s), 1665 (s), 1624 (s); ${ }^{1} \mathrm{H}$ NMR (DMSO- $\left.d_{6}, 400 \mathrm{MHz}\right) \delta /$ ppm : $0.74\left(3 \mathrm{H}, \mathrm{s}, \mathrm{CH}_{3}\right), 0.81\left(3 \mathrm{H}, \mathrm{s}, \mathrm{CH}_{3}\right), 1.75-1.93$ $\left(2 \mathrm{H}, \mathrm{m}, \mathrm{CH}_{2}\right), 2.09-2.24\left(2 \mathrm{H}, \mathrm{m}, \mathrm{CH}_{2}\right), 5.34(1 \mathrm{H}, \mathrm{s}, \mathrm{CH})$, 7.05-7.18 (4H, m, Arom), 7.50 (1H, s, NH), $9.33(1 \mathrm{H}, \mathrm{s}$, $\mathrm{NH})$ ppm; ${ }^{13} \mathrm{C}$ NMR (DMSO- $\left.d_{6}, 100 \mathrm{MHz}\right) \delta / \mathrm{ppm}$ : $28.64\left(\mathrm{CH}_{3}\right), 30.33(\mathrm{C}), 33.85\left(\mathrm{CH}_{2}\right), 51.38(\mathrm{CH}), 107.41$ (C), $128.99(\mathrm{CH}), 130.55(\mathrm{CH}), 131.01(\mathrm{CH}), 133.46$ $(\mathrm{CH}), 142.79(\mathrm{CH}), 154.66(\mathrm{C}), 161.84(\mathrm{CO}), 194.24$ (CO) ppm; Anal. Calcd. for $\mathrm{C}_{16} \mathrm{H}_{17} \mathrm{ClN}_{2} \mathrm{O}_{2}: \mathrm{C}, 63.05 ; \mathrm{H}$, 5.62; N, $9.19 \%$. Found: C, 63.26; H, 5.37; N, $9.12 \%$.

4h: IR (KBr) $v_{\max } / \mathrm{cm}^{-1}: 3285$ (s), 3190 (s), 1646 (s), 1605 (s); ${ }^{1} \mathrm{H}$ NMR (DMSO- $\left.d_{6}, 400 \mathrm{MHz}\right) \delta / \mathrm{ppm}: 0.79$ $\left(3 \mathrm{H}, \mathrm{s}, \mathrm{CH}_{3}\right), 0.88\left(3 \mathrm{H}, \mathrm{s}, \mathrm{CH}_{3}\right), 1.93-1.97(2 \mathrm{H}, \mathrm{d}, J=$ $\left.16 \mathrm{~Hz}, \mathrm{CH}_{2}\right), 2.00-2.04\left(2 \mathrm{H}, \mathrm{d}, J=16 \mathrm{~Hz}, \mathrm{CH}_{2}\right), 2.26$ $\left(1 \mathrm{H}, \mathrm{s}, \mathrm{CH}_{3}\right), 4.50(1 \mathrm{H}, \mathrm{s}, \mathrm{CH}), 6.80-6.98(4 \mathrm{H}, \mathrm{m}$, Arom), 7.06 (2H, br, NH) ppm; Anal. Calcd. for
$\mathrm{C}_{17} \mathrm{H}_{20} \mathrm{~N}_{2} \mathrm{O}_{2}$ : C, 71.81; H, 7.09; N, $9.85 \%$. Found: C, 72.03; H, 6.90; N, $9.72 \%$.

4j: IR (KBr) $v_{\max } / \mathrm{cm}^{-1}: 3228$ (s), 2962 (s), 1649 (s), 1620 (s); ${ }^{1} \mathrm{H}$ NMR (DMSO- $\left.d_{6}, 400 \mathrm{MHz}\right) \delta / \mathrm{ppm}$ : 1.83-1.89 (2H, m, $\left.\mathrm{CH}_{2}\right), 1.94-2.00\left(2 \mathrm{H}, \mathrm{m}, \mathrm{CH}_{2}\right)$, 2.306-2.347 (2H, m, $\left.\mathrm{CH}_{2}\right), 4.594(1 \mathrm{H}, \mathrm{s}, \mathrm{CH})$, 7.096-7.241 (5H, m, Arom), 7.516 (1H, s, NH), 9.347 $(1 \mathrm{H}, \mathrm{s}, \mathrm{NH}) \mathrm{ppm} ;{ }^{13} \mathrm{C}$ NMR (DMSO- $\left.d_{6}, 100 \mathrm{MHz}\right) \delta /$ ppm: $21.00\left(\mathrm{CH}_{2}\right), 27.77\left(\mathrm{CH}_{2}\right), 36.75\left(\mathrm{CH}_{2}\right), 50.94$ (CH), $107.69(\mathrm{C}), 113.39(\mathrm{CH}), 129.03(\mathrm{CH}), 136.07$ (C), 149.51 (C), 154.81 (C), 158.74 (CO), 191.82 (CO) ppm; Anal. Calcd. for $\mathrm{C}_{14} \mathrm{H}_{14} \mathrm{~N}_{2} \mathrm{O}_{2}$ : C, 69.41; H, 5.82; N, $11.56 \%$. Found: C, 69.22; H, 5.92; N, $11.41 \%$.

4n: IR (KBr) $v_{\max } / \mathrm{cm}^{-1}: 3302$ (s), 3190 (s), 1688 (s), 1667 (s), 1636 (s); ${ }^{1} \mathrm{H}$ NMR (DMSO- $d_{6}, 400 \mathrm{MHz}$ ) $\delta /$ ppm : $1.35-1.61\left(2 \mathrm{H}, \mathrm{m}, \mathrm{CH}_{2}\right), 1.74-1.91\left(2 \mathrm{H}, \mathrm{m}, \mathrm{CH}_{2}\right)$, 1.96-2.18 (2H, m, $\left.\mathrm{CH}_{2}\right), 3.50\left(1 \mathrm{H}, \mathrm{s}, \mathrm{OCH}_{3}\right), 4.39(1 \mathrm{H}$, s, CH), 6.56-6.65 (4H, m, Arom), $6.96(1 \mathrm{H}, \mathrm{s}, \mathrm{NH})$, $8.11(1 \mathrm{H}, \mathrm{s}, \mathrm{NH}) \mathrm{ppm} ;{ }^{13} \mathrm{C} \mathrm{NMR}\left(\mathrm{DMSO}-d_{6}, 100 \mathrm{MHz}\right)$ $\delta$ / ppm: $20.99\left(\mathrm{CH}_{2}\right), 29.00\left(\mathrm{CH}_{2}\right), 37.27(\mathrm{CH} 2)$, $55.02(\mathrm{CH}), 101.62(\mathrm{C}), 110.42(\mathrm{CH}), 111.48(\mathrm{CH})$, $119.84(\mathrm{CH}), 126.56(\mathrm{CH}), 129.25(\mathrm{CH}), 131.70(\mathrm{CH})$, 156.55 (C), 169.11 (CO), 196.02 (CO) ppm; Anal. Calcd. for $\mathrm{C}_{15} \mathrm{H}_{16} \mathrm{~N}_{2} \mathrm{O}_{3}$ : C, 66.16; H, 5.92; N, $10.29 \%$. Found: C, 66.40; H, 5.752; N, 10.11\%.

4q: $\operatorname{IR}(\mathrm{KBr}) v_{\max } / \mathrm{cm}^{-1}: 3437$ (s), 3305 (s), 1664 (s), 1613 (s), 1589 (s); ${ }^{1} \mathrm{H}$ NMR (DMSO-d $\left.6,400 \mathrm{MHz}\right) \delta /$ ppm : $0.679\left(3 \mathrm{H}, \mathrm{s}, \mathrm{CH}_{3}\right), 0.803\left(3 \mathrm{H}, \mathrm{s}, \mathrm{CH}_{3}\right), 1.82-2.01$ $\left(2 \mathrm{H}, \mathrm{m}, \mathrm{CH}_{2}\right), 2.06-2.22\left(2 \mathrm{H}, \mathrm{m}, \mathrm{CH}_{2}\right), 4.94(1 \mathrm{H}, \mathrm{s}$, $\mathrm{CH}), 7.06-7.16$ (4H, m, Arom), 7.61 (1H, s, NH), 9.35 $(1 \mathrm{H}, \mathrm{s}, \mathrm{NH}) \mathrm{ppm} ;{ }^{13} \mathrm{C}$ NMR (DMSO- $\left.d_{6}, 100 \mathrm{MHz}\right) \delta /$ ppm: $27.28\left(\mathrm{CH}_{3}\right), 29.12(\mathrm{C}), 32.78\left(\mathrm{CH}_{2}\right), 50.21\left(\mathrm{CH}_{2}\right)$, $52.08(\mathrm{CH}), 107.25(\mathrm{C}), 125.32(\mathrm{CH}), 126.25(\mathrm{CH})$, $127.35(\mathrm{CH}), 130.48(\mathrm{CH}), 133.26(\mathrm{CH}), 145.90(\mathrm{CH})$, 147.42 (C), 158.11 (CO), 193.03 (CO) ppm; Anal. Calcd. for $\mathrm{C}_{16} \mathrm{H}_{17} \mathrm{ClN}_{2} \mathrm{O}_{2}$ : C, 63.05; H, 5.62; N, $9.19 \%$. Found: C, 63.22; H, 5.55; N, $9.08 \%$.

4r: IR (KBr) $v_{\max } / \mathrm{cm}^{-1}: 3278(\mathrm{~s}), 3162(\mathrm{~s}), 1642$ (s), 1572 (s); ${ }^{1} \mathrm{H}$ NMR (DMSO-d $\left.6,400 \mathrm{MHz}\right) \delta / \mathrm{ppm}$ : $0.903\left(3 \mathrm{H}, \mathrm{s}, \mathrm{CH}_{3}\right), 1.041\left(3 \mathrm{H}, \mathrm{s}, \mathrm{CH}_{3}\right), 2.093-2.053$ $\left(2 \mathrm{H}, \mathrm{m}, \mathrm{CH}_{2}\right), 2.212\left(2 \mathrm{H}, \mathrm{s}, \mathrm{CH}_{2}\right), 2.25\left(3 \mathrm{H}, \mathrm{s}, \mathrm{CH}_{3}\right), 5.2$ (1H, s, CH), 7.10-7.15 (m, 4H, Arom), 9.65 (1H, s, NH), $10.56(1 \mathrm{H}, \mathrm{s}, \mathrm{NH}) \mathrm{ppm} ;{ }^{13} \mathrm{C} \mathrm{NMR}\left(\mathrm{DMSO}_{6}, 100 \mathrm{MHz}\right)$ $\delta$ / ppm : $20.33\left(\mathrm{CH}_{3}\right), 27.34\left(\mathrm{CH}_{3}\right), 29.60\left(\mathrm{CH}_{3}\right), 32.75$ (C), $37.84(\mathrm{CH} 2), 50.75\left(\mathrm{CH}_{2}\right), 51.51(\mathrm{CH}), 105.84(\mathrm{C})$, $123.29(\mathrm{CH}), 129.00(\mathrm{CH}), 137.37(\mathrm{C}), 140.00(\mathrm{C})$, 149.47 (C), 174.61 (CS), 195.06 (CO) ppm; Anal. Calcd. for $\mathrm{C}_{17} \mathrm{H}_{20} \mathrm{~N}_{2} \mathrm{OS}$ : C, 67.97; H, 6.71; N, 9.32; S, $10.67 \%$. Found: C, 68.18; H, 6.50; N, 9.21; S, $10.45 \%$.

4t: IR (KBr) $v_{\max } / \mathrm{cm}^{-1}: 3262$ (s), 3165 (s), 1666 (s), 1641 (s), 1584 (s); ${ }^{1} \mathrm{H}$ NMR (DMSO-d, $\left.400 \mathrm{MHz}\right) \delta /$ ppm : $0.909\left(3 \mathrm{H}, \mathrm{s}, \mathrm{CH}_{3}\right), 1.033\left(3 \mathrm{H}, \mathrm{s}, \mathrm{CH}_{3}\right), 2.09-2.22$ $\left(2 \mathrm{H}, \mathrm{m}, \mathrm{CH}_{2}\right), 2.34-2.43\left(2 \mathrm{H}, \mathrm{m}, \mathrm{CH}_{2}\right), 3.73(3 \mathrm{H}, \mathrm{s}$, 
$\left.\mathrm{OCH}_{3}\right), 5.12(1 \mathrm{H}, \mathrm{s}, \mathrm{CH}), 6.896 .91(2 \mathrm{H}, \mathrm{m}$, Arom), 7.14-7.12 (2H, m, Arom), 9.64 (1H, s, NH), $10.54(1 \mathrm{H}$, $\mathrm{s}, \mathrm{NH}) \mathrm{ppm} ;{ }^{13} \mathrm{C}$ NMR (DMSO- $\left.d_{6}, 100 \mathrm{MHz}\right) \delta / \mathrm{ppm}$ : $27.25\left(\mathrm{CH}_{3}\right), 29.29\left(\mathrm{CH}_{3}\right), 32.73(\mathrm{C}), 38.93\left(\mathrm{CH}_{2}\right)$, $50.31\left(\mathrm{CH}_{2}\right), 52.09(\mathrm{CH}), 55.56\left(\mathrm{OCH}_{3}\right), 108.75(\mathrm{C})$, $114.27(\mathrm{CH}), 128.09(\mathrm{CH}), 136.06(\mathrm{C}), 148.92(\mathrm{C})$, 159.09 (C), 174.82 (CS) , 194.11 (CO) ppm; Anal. Calcd. for $\mathrm{C}_{17} \mathrm{H}_{20} \mathrm{~N}_{2} \mathrm{O}_{2} \mathrm{~S}$ : C, 64.53; H, 6.37; N, 8.85; S, $10.13 \%$. Found: C, 64.61; H, 6.39; N, 8.72; S, $9.90 \%$.

4u: IR (KBr) $v_{\max } / \mathrm{cm}^{-1}: 3262$ (s), 3173 (s), 1698 (s), 1620 (s), 1567 (s); ${ }^{1} \mathrm{H}$ NMR (DMSO- $\left.d_{6}, 400 \mathrm{MHz}\right) \delta /$ ppm : $0.897\left(3 \mathrm{H}, \mathrm{s}, \mathrm{CH}_{3}\right), 1.035\left(3 \mathrm{H}, \mathrm{s}, \mathrm{CH}_{3}\right), 2.06-2.23$ $\left(2 \mathrm{H}, \mathrm{m}, \mathrm{CH}_{2}\right), 2.36-2.45\left(2 \mathrm{H}, \mathrm{m}, \mathrm{CH}_{2}\right), 5.12(1 \mathrm{H}, \mathrm{s}$, $\mathrm{CH}), 7.22-7.28(\mathrm{~m}, 2 \mathrm{H}$, Arom), 7.33-7.36 (2H, m, Arom), 9.69 (1H, s, NH), $10.59(1 \mathrm{H}, \mathrm{s}, \mathrm{NH}) \mathrm{ppm} ;{ }^{13} \mathrm{C}$ NMR (DMSO- $\left.d_{6}, 100 \mathrm{MHz}\right) \delta / \mathrm{ppm}: 27.24\left(\mathrm{CH}_{3}\right)$, $29.28(\mathrm{C}), 32.73\left(\mathrm{CH}_{2}\right), 50.31\left(\mathrm{CH}_{2}\right), 52.69(\mathrm{CH}), 108.61$ (C), $126.89(\mathrm{CH}), 128.02(\mathrm{CH}), 128.96(\mathrm{CH}), 143.83$ (CH), 149.16 (C), 175.08 (CS), 194.12 (CO) ppm; Anal. Calcd. for $\mathrm{C}_{16} \mathrm{H}_{18} \mathrm{~N}_{2} \mathrm{OS}$ : C, 67.10; H, 6.33; N, 9.78; S, $11.20 \%$. Found: C, 67.22; H, 6.40; N, 9.65; S, $11.35 \%$.

\section{CONCLUSION}

In this study, a rapid and modified method for the one-pot reaction of aldehydes, cyclic 1,3-dione and urea/thiourea by employing the $\operatorname{In}(\mathrm{OTf})_{3}$ as a commercially available catalyst under solvent-free conditions has been described. This simple catalytic system is remarkably tolerant to a variety of functional groups on the arylaldehyde and offers significant advantages such as, low catalyst loading, high yields, reducing the use of organic solvents, short reaction times and operational simplicity. Therefore, in employing a small amount of safe, inexpensive, and powerful catalyst under solvent-free conditions, this protocol is economic and eco-friendly.

Supplementary Materials. - Supporting informations to the paper are enclosed to the electronic version of the article. These data can be found on the website of Croatica Chemica Acta (http://public.carnet.hr/ccacaa).

Acknowledgements. We are thankful to the Islamic Azad University of Gachsaran for partial support of this work.

\section{REFERENCES}

1. R. Karimian, F. Piri, B. Karimi, and A. Moghimi, Croat. Chem. Acta 84 (2011) 111.
2. E. J. Lenardao, D. O. Trecha, P. d. C. Ferreira, R. G. Jacob, and Gelson Perin, J. Braz. Chem. Soc. 20 (2009) 93.

3. V. Maslak, R. Matovic, and R. N. Saicic, Tetrahedron Lett. 43 (2002) 5411.

4. A. V. Ivachtchenko, Y. A. Ivanenkov, V. M. Kysil, M. Y. Krasavin, and A. P. Ilyin, Russ. Chem. Rev. 79 (2010) 787.

5. A. Shaabani, A. Rahmati, A. H. Rezayan, and H. R. Khavasi, J. Iran. Chem. Soc. 8 (2001) 24.

6. K. Chen, K. Wang, A. M. Kirichian, A. F. Al Aowad, L. K. Iyer, S. J. Adelstein, and A. I. Kassism, Mol. Cancer Ther. 5 (2006) 3001 .

7. A. Mobinikhaledi, N. Foroughifar, H. Khodaei, Eur. J. Chem. 4 (2010) 291.

8. W. Ried, W. Stephan, Chem Ber. 95 (1962) 3042.

9. H. Kotsuki, H. Sakai, H. Morimoto, H. Svenaga. Syn Lett. 12 (1999) 1993

10. P. Jones, M. Chambers, Tetrahedron 58 (2002) 9973.

11. Z. Hasani, M. Islami, M. Kalantari, Bio Medicinal Chem. 16 (2006) 4479

12. S. Kirti, B. Bapurao, S.Murliduar, Tetrahedron 51(2010) 3616.

13. S. Karami, B. Karami, S. Khodabakhshi, J. Chin. Chem. Soc. 60 (2013) 22

14. (a) K. S. Niralwad, B. B. Shingate, M. S. Shingare, Tetrahedron Lett. 51 (2010) 3616; (b) M. Kidwai, S. Saxena, M. K. Rahman Khan, S. S. Thukral, Eur. J. Med. Chem. 40 (2005) 816; (c) R. Ghosh, S. Maiti, A. Chakraborty, J. Mol. Catal. A: Chemical 217 (2004) 47; (d) P. Shanmugam, G. Annie and P. T . Perumal, J. Heterocyclic Chem. 40, 879 (2003); (e) Suresh, J. S. Sandhu, Arkivoc i (2012) 66; (f) S. Kantevari, R. Bantu, L. Nagarapu, Arkivoc xvi (2006) 136; (g) C. O. Kappe, J. Org. Chem. 62 (1997) 7201; (h) A. Dondoni, A. Massi, E. Minghini, S. Sabbatini, and V. Bertolasi, J. Org. Chem. 68 (2003) 6172; (i) A. Mobinikhaledi, N. Foroughifar, and G. Karimi, Synth. React. Inorg. M. 37 (2007) 279; (j) M. Zendehdel, A. Mobinikhaledi, and A. Asgari, J. Incl. Phenom. Macro. 60 (2008) 353; (k) C. J. Liu, and J. D. Wang, Molecules 14 (2009) 763; (1) M. Tajbakhsh, B. Mohajerani, M. M. Heravi, and A. N. Ahmadi, J. Mol. Catal. A Chem. 236 (2005) 216; (m) A. Mobinikhaledi, N. Foroughifar, and H. Khodaei, Eur. J. Chem. 1 (2010) 291. (n) V. S. Palekar, S. R. Shukla, Green Chem. Lett. Rev. 1 (2008) 185.

15. J. Dai, J. Wu, G. Zhao, and W-M. Da, Chem. Eur. J. 17 (2011) 8290.

16. R. Ghosh, S. Maiti, A. Chakraborty, and R. Halder, J. Mol. Catal. A Chem. 215 (2004) 49.

17. K. C. Lekhok, D. Prajapati, and R. C. Boruah, Synlett (2008) 655.

18. S. K. Giri, M. Verma, and K. P. R. Kartha, J. Carbohydr. Chem. 27 (2008) 464.

19. C. G. Frost, J. P. Hartley, and A. J. Whittle, Synlett (2001) 830.

20. B. Karami, and S. Khodabakhshi, J. Serb. Chem. Soc. 76 (2011) 1191.

21. B. Karami, M. Taei, S. Khodabakhshi, and M. Jamshidi, J. Sulf. Chem. 33 (2012) 65

22. B. Karami, S. Khodabakhshi, and M. Nikrooz, J. Chin. Chem. Soc. 58 (2011) 1.

23. B. Karami, S. Khodabakhshi, and M. Nikrooz, Polycyclic Aromat. Comp. 97 (2011) 31.

24. A. Shaabani, A. Sarvary, A. Rahmati, and A. H. Rezayan, Lett. Org. Chem. 4 (2007) 68. 